\title{
An Exact Solution to the Quadratic Damping Strong Nonlinearity Duffing Oscillator
}

\author{
Alvaro H. Salas $\mathbb{D}^{1},{ }^{1}$ S. A. El-Tantawy $\mathbb{D}^{2,3}$ and Noufe H. Aljahdaly ${ }^{4}{ }^{4}$ \\ ${ }^{1}$ Department of Mathematics, Universidad Nacional de Colombia, \\ Derpartment of Mathematics and Statistics FIZMAKO Research Group, Nubia Campus, Manizales, Colombia \\ ${ }^{2}$ Department of Physics, Faculty of Science, Port Said University, Port Said 42521, Egypt \\ ${ }^{3}$ Research Center for Physics (RCP), Department of Physics, Faculty of Science and Arts, Al-Mikhwah, Al-Baha University, \\ Al-Baha, Saudi Arabia \\ ${ }^{4}$ Department of Mathematics, Faculty of Sciences and Arts-Rabigh Campus, King Abdulaziz University, Rabigh 21911, \\ Jeddah, Saudi Arabia \\ Correspondence should be addressed to S. A. El-Tantawy; samireltantawy@yahoo.com
}

Received 31 August 2020; Revised 28 December 2020; Accepted 31 December 2020; Published 19 January 2021

Academic Editor: Kai Zhang

Copyright (c) 2021 Alvaro H. Salas et al. This is an open access article distributed under the Creative Commons Attribution License, which permits unrestricted use, distribution, and reproduction in any medium, provided the original work is properly cited.

\begin{abstract}
The nonlinear equations of motion such as the Duffing oscillator equation and its family are seldom addressed in intermediate instruction in classical dynamics; this one is problematic because it cannot be solved in terms of elementary functions before. Thus, in this work, the stability analysis of quadratic damping higher-order nonlinearity Duffing oscillator is investigated. Hereinafter, some new analytical solutions to the undamped higher-order nonlinearity Duffing oscillator in the form of Weierstrass elliptic function are obtained. Posteriorly, a novel exact analytical solution to the quadratic damping higher-order nonlinearity Duffing equation under a certain condition (not arbitrary initial conditions) and in the form of Weierstrass elliptic function is derived in detail for the first time. Furthermore, the obtained solutions are camped to the Runge-Kutta fourth-order (RK4) numerical solution.
\end{abstract}

\section{Introduction}

In the last few decades, considerable work has been invested in developing new methods for analytical and numerical solutions for several differential equations [1-4] such as strongly nonlinear oscillators, but it is still difficult to obtain convergent results in cases of strong nonlinearity. The Duffing oscillator model is one of the most important and most popular models in dynamic systems due to its importance in explaining many nonlinear phenomena in science and engineering. For instance, the nonlinear Duffing equation was devoted for studying the dynamical behavior of oscillations in plasma physics [5], plasma physics and rigid rotator [6], magneto-elastic mechanical systems [7], nonlinear vibration of beams and plates [8], fluid flow induced vibration [9], large amplitude oscillation of centrifugal governor systems [10], etc. Since the early century so far, a lot of researchers have been devoted carefully to study the Duffing oscillator model in several physical situations [11]. So far the Duffing oscillator model has been an effective and applicable example to nonlinear oscillations in dynamic systems [12]. In addition to its mentioned applications, it also has various applications in the mechanical engineering $[9,10]$ and electrical engineering $[13,14]$. It has been solved numerically and analytically via using various methods in order to recognize its physical characters. Some methods which consider the conservative Duffing osciullators are the harmonic balance technique [15], the frequency-amplitude formulation [16], the homotopy perturbation method $[10,17]$, the coupled homotopy-variational approach [18], and the differential transform [19]. It is known that if the damping effect is taken into account in the conservative 
Duffing oscillators, the model becomes a nonconservative system and the oscillation amplitude reduces over time. There are many methods that have been devoted to solve the nonconservative Duffing oscillators, whether they are semianalytical or numerical solutions [20-26]. However, most of the analytical methods are unable to deal with the Duffing equation in the presence of a damping effect. Therefore, all published solutions are either approximate numerical solutions, semianalytical or approximate analytical solutions, or analytical solutions under certain restrictions [20-31].

The most popular formula for conservative Duffing oscillator for arbitrary initial conditions is given by $[32,33]$

$$
\left\{\ddot{x}(t)+p x(t)+q x^{3}(t)=0, x(0)=x_{0}, x^{\prime}(0)=\dot{x}_{0} .\right.
$$

This equation is completely integrable, and its exact analytical solutions in the Jacobi elliptic function forms have been obtained [32, 33]. However, equation (1) without damping effect is not realistic physically, and if the damping effect is taken into account, the following nonconservative Duffing oscillator for arbitrary initial conditions may be obtained as $[5,6,34-37]$

$$
\left\{\ddot{x}+\varepsilon \dot{x}+p x+q x^{3}=0, x(0)=x_{0} \& x^{\prime}(0)=\dot{x}_{0} .\right.
$$

In generally, equation (2) is not integrable differential equation due to the presence damping term $(\varepsilon \dot{x})$, and many methods have been devoted to solve this equation, as was previously mentioned in all the upper references such as [5, 6, 34-37].

The generalized form of the Duffing oscillator equation which has been modelled in an arbitrary form of the nonlinear differential equation may be written as follows:

$$
\ddot{x}+\varepsilon \dot{x}|\dot{x}|^{p-1}+f(x)=0 .
$$

In equation (3), the second term $\dot{x}|\dot{x}|^{p^{-1}}$ refers to the nonlinear damping term which is proportional to the $p$ th power of $\dot{x}$ and $\varepsilon$ is a positive or negative damping parameter where its sign depends on the type of the physical application. The third term in equation (3), i.e., $f(x)=\sum_{j=1}^{n} a_{n} x^{n}$ involves the linear term $\left(a_{1} x\right)$ and the nonlinear terms of $x$ which is known as the restoring force of the dynamical system. As we mentioned earlier, many authors studied the linear damping $(p=1)$ for linear and cubic restoring forces $x$ and $x^{3}[5,6,34-37]$. On the contrary, there are a few attempts to solve and investigate the damping Duffing oscillator for quadratic restoring force $(p=2)$ [38-40]. The elliptic-harmonic balance method has been applied to find an approximate analytical solution in the Jacobi elliptic function form to a quadratic damping system with linear restoring force: $\ddot{x}+\varepsilon \dot{x}^{2}+\alpha x=0$ [38]. In [40], some solutions to the quadratic damping Helmholtz-Duffing equation $\left(\ddot{x}+\varepsilon \dot{x}^{2}+\alpha x+\beta x^{2}+\gamma x^{3}=0\right)$ in the form of classical Jacobi elliptic functions have been discussed in details. Given the difficulty of solving quadratic damping Duffing equation with higher-order nonlinearity, this problem remains one of the most important hot topics that require a more careful study to obtain more accurate solutions.

In this paper, the quadratic damping Duffing oscillator with linear and cubic restoring forces $\left(f(x)=p x+q x^{3}\right)$ is considered under the initial conditions $x(0)=x_{0}$ and $\dot{x}(0)=\dot{x}_{0}$. Thus, the mathematical model of this physical situation may be written as the initial value problem (IVP):

$$
\left\{\ddot{x}+\varepsilon \dot{x}^{2}+p x+q x^{3}=0, x(0)=x_{0} \& x^{\prime}(0)=\dot{x}_{0} .\right.
$$

The IVP (4) is considered a nonintegrable differential equation but may admit exact analytical solution under a certain condition. In this study, we will derive this condition in detail in order to find an exact solution to the IVP (4) in terms of the Weierstrass elliptic function for the first time. Moreover, before finding the exact solution to the IVP (4), we will study its stability in order to analyze its characteristics to recognize its dynamical behavior.

The rest of the paper is presented in the following points. The stability analysis of the quadratic damping higher-order nonlinearity Duffing equation will be investigated in Section 2. Some possible analytical solutions to the Duffing equation and its family in the form of Jacobi elliptic function and Weierstrass elliptic function will be discussed in detail. Firstly, we will discuss some possible solutions to the IVP (2) in terms of Jacobi elliptic function depending on the discriminant of the IVP (2). Thereafter, the exact analytical solutions to both the IVP (2) and IVP (4) in terms of Weierstrass elliptic function will be derived and obtained in details. The summary of our investigations will be introduced in Section 4.

\section{Stability Analysis to the Quadratic Damping Duffing Equation}

The first equation in the IVP (4) may be written as a dynamical system as follows:

$$
\left\{\begin{array}{l}
\dot{x}=f(x, y)=y, \\
\dot{y}=g(x, y)=-\varepsilon y^{2}-p x-q x^{3} .
\end{array}\right.
$$

The first integral reads

$$
\frac{e^{2 \varepsilon x}}{4 \varepsilon^{4}}\left(\begin{array}{c}
-2 \varepsilon^{2} p+4 \varepsilon^{3} p x+4 \varepsilon^{3} q x^{3} \\
-6 \varepsilon^{2} q x^{2}+6 \varepsilon q x-3 q+4 \varepsilon^{4} y^{2}
\end{array}\right)=h,
$$

System (5) becomes Hamilton if the following condition is fulfilled

$$
\partial_{x} f(x, y)+\partial_{y} g(x, y)=0
$$

with

$$
\left\{\begin{array}{l}
f(x, y)=\partial_{y} h(x, y), \\
g(x, y)=-\partial_{x} h(x, y),
\end{array}\right.
$$

It is noted that condition (7) is fulfilled; thus, system (5) becomes Hamilton with the following Hamiltonian: 


$$
H(x, y)=\frac{e^{2 \varepsilon x}}{4 \varepsilon^{4}}\left(\begin{array}{c}
-2 \varepsilon^{2} p+4 \varepsilon^{3} p x+4 \varepsilon^{3} q x^{3} \\
-6 \varepsilon^{2} q x^{2}+6 \varepsilon q x-3 q+4 \varepsilon^{4} y^{2}
\end{array}\right) .
$$

Now, if $p q<0$, then system (5) has three equilibrium points:

$$
\begin{aligned}
& \left(x_{1}, y_{1}\right)=(0,0), \\
& \left(x_{2}, y_{2}\right)=\left(\sqrt{\frac{-p}{q}}, 0\right), \\
& \left(x_{3}, y_{3}\right)=\left(-\sqrt{\frac{-p}{q}}, 0\right),
\end{aligned}
$$

and, for $p q>0$, there is only one equilibrium point:

$$
\left(x_{1}, y_{1}\right)=(0,0) \text {. }
$$

The Jacobian matrix for system (5) at equilibrium point $\left(x_{e}, y_{e}\right)$ reads

$$
A\left(x_{e}, y_{e}\right)=\left[\begin{array}{cc}
0 & -p-3 q x_{e}^{2} \\
1 & -2 y_{e} \varepsilon
\end{array}\right] .
$$

Thus, the determinant and trace of the Jacobian matrix $A\left(x_{e}, y_{e}\right)$ may be estimated as

$$
\left.\begin{array}{l}
\operatorname{Det}\left(A\left(x_{e}, y_{e}\right)\right)=p+3 q x_{e}^{2}, \\
\operatorname{Tr}\left(A\left(x_{e}, y_{e}\right)\right)=-2 \varepsilon y_{e} .
\end{array}\right\}
$$

The behavior of the dynamical system near the equilibria is described by the Poincaré Diagram as given in [41].

Let us analyze the equilibrium points.

For $\left(x_{e}, y_{e}\right)=(0,0)$, we obtain

$$
\left.\begin{array}{l}
\operatorname{Det}(A(0,0))=p \\
\operatorname{Tr}(A(0,0))=0 .
\end{array}\right\}
$$

The origin becomes a central point if $p>0$ and a saddle if $p<0$, as shown in Figures 1(a) and 1(b), respectively.

For $p q<0$ and $\left(x_{e}, y_{e}\right)=(\sqrt{(-p / q)}, 0)$ or $\left(x_{e}, y_{e}\right)=(-\sqrt{(-p / q)}, 0)$, we obtain

$$
\left.\begin{array}{l}
\operatorname{Det}\left(A\left(x_{e}, y_{e}\right)\right)=-2 p \\
\operatorname{Tr}\left(A\left(x_{e}, y_{e}\right)\right)=0
\end{array}\right\}
$$

It is clear that if $p<0$, the equilibrium point becomes a central point and a saddle point is covered if $p>0$, as illustrated in Figures 2(a) and 2(b), respectively.

\section{Analytical Solutions to the Quadratic Damping Duffing Equation}

Before embarking on solving the IVP (4), we will discuss some possible solutions to both the IVP (1) and the IVP (2) in terms of Weierstrass elliptic function.
3.1. The Analytical Solution of Undamping Duffing Equation in Terms of Weierstrass Elliptic Function. Let us solve the following undamped IVP:

$$
\left\{\begin{array}{l}
x^{\prime \prime}(t)+p x(t)+q x^{3}(t)=0, \\
x(0)=x_{0} \& x^{\prime}(0)=\dot{x}_{0},
\end{array}\right.
$$

where $\left(x_{0}^{2}+\dot{x}_{0}^{2}\right) q \neq 0$, and the primes represent the differentiation with respect to the independent variable say " $t$." Here, it is assumed that $p, q, u_{0}$, and $\dot{u}_{0}$ are real numbers. To find an analytical solution to the IVP (16) in the form of Weierstrass elliptic function $\wp$, the following ansatz is assumed:

$$
x(t)=A+\frac{B}{1+C \wp\left(t+t_{0} ; g_{2}, g_{3}\right)},
$$

where $A, B, C, g_{2}, g_{3}$, and $t_{0}$ are some constants to be determined later and the condition $\left(p+q x_{0}^{2}\right)^{2}+2 q \dot{x}_{0}^{2}>0$ must be fulfilled. Note that the Weierstrass elliptic function $\wp \equiv \wp\left(t+t_{0} ; g_{2}, g_{3}\right) \equiv \wp\left(z ; g_{2}, g_{3}\right)$ satisfies the following relations:

$$
\left\{\begin{array}{l}
\left(\wp^{\prime}\right)^{2}=4 \wp^{3}-g_{2} \wp-g_{3} \\
\wp^{\prime \prime}=-\frac{g_{2}}{2}+6 \wp^{2}
\end{array}\right.
$$

Substituting both equations (17) and (18) into equation (16) and after some simplifications, we finally obtain

$$
\frac{1}{2(1+C \wp)} \sum_{j=0}^{3} \mathbb{R}_{j} \wp^{j}=0,
$$

where the values of the coefficients $\mathbb{R}_{j}$ are given in Appendix A. Equating the coefficients $\mathbb{R}_{j}$ to zero, we get a system of algebraic equations, and by solving this system, we obtain

$$
\begin{aligned}
B & =-A\left(2+\frac{4 p}{3 A^{2} q+p}\right), \\
C & =\frac{12}{3 A^{2} q+p}, \\
g_{2} & =\frac{1}{12}\left(-3 A^{4} q^{2}-6 A^{2} p q+p^{2}\right), \\
g_{3} & =\frac{1}{216} p\left(9 A^{4} q^{2}+18 A^{2} p q+p^{2}\right) .
\end{aligned}
$$

Thus, the general exact solution to the undamping strong nonlinear Duffing equation $\left(x^{\prime \prime}(t)+p x(t)+q x^{3}(t)=0\right)$ in terms of Weierstrass elliptic function is given by

$$
x(t)=A-\frac{6 A\left(A^{2} q+p\right)}{3 A^{2} q+p+12 \wp\left(\begin{array}{c}
t+t_{0} ;(1 / 12)\left(-3 q^{2} A^{4}-6 p q A^{2}+p^{2}\right) \\
(1 / 216) p\left(9 q^{2} A^{4}+18 p q A^{2}+p^{2}\right)
\end{array}\right) .}
$$

Inserting the initial conditions $x(0)=x_{0}$ and $x^{\prime}(0)=\dot{x}_{0}$ into solution (21), we finally get the value of $t_{0}$ as follows: 


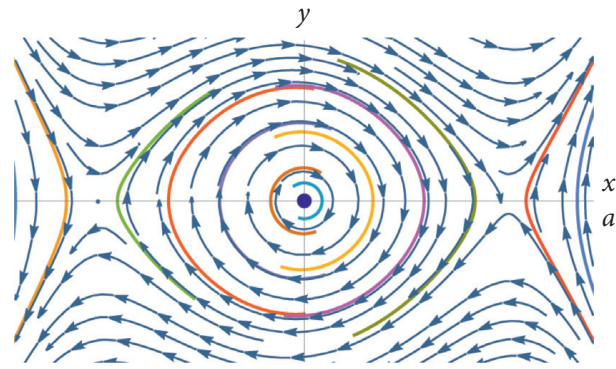

(a)

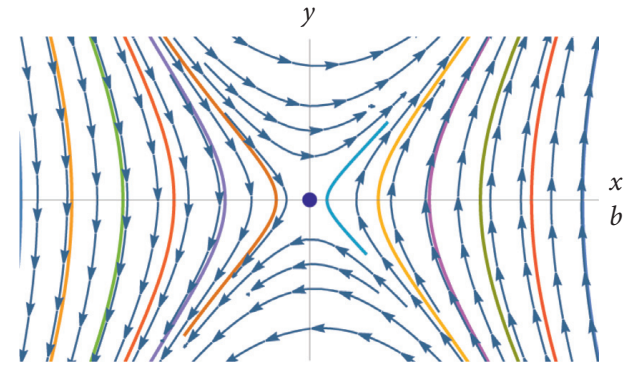

(b)

Figure 1: Phase Portrait of system (5) for the equilibrium point $\left(x_{e}, y_{e}\right)=(0,0)$ : (a) for central point $(p>0)$ where $p=1, q=-2$, and $\varepsilon=0.2$ and (b) for saddle point $(p<0)$ where $p=-1, q=-2$, and $\varepsilon=0.2$.

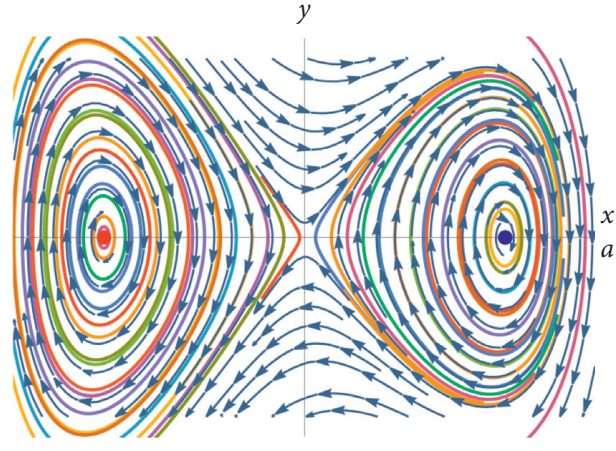

(a)

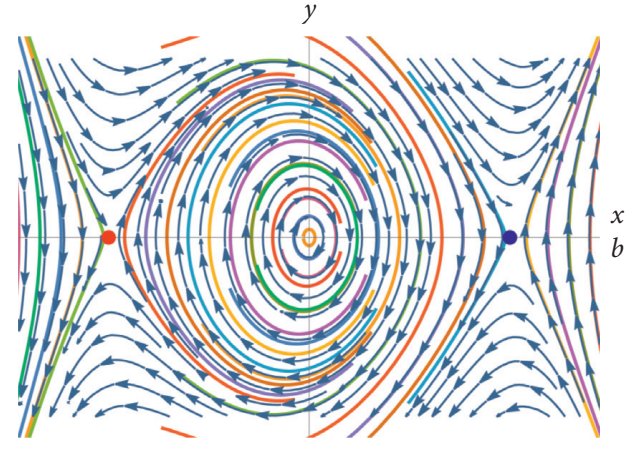

(b)

Figure 2: Phase Portrait of system (5) for the equilibrium points $\left(x_{e}, y_{e}\right)=(\sqrt{(-p / q)}, 0)$ or $\left(x_{e}, y_{e}\right)=(-\sqrt{(-p / q)}, 0)$ for $p q<0$ : $($ a) for central point $(p<0)$ where $p=-2, q=0.4$, and $\varepsilon=0.2$ and (b) for saddle point $(p>0)$ where $p=2, q=-0.4$, and $\varepsilon=0.2$.

$$
t_{0}= \pm \wp^{-1}\left(\frac{x_{0}-A-B}{C\left(A-x_{0}\right)} ; g_{2}, g_{3}\right) \text {, }
$$

and the value of $A$ may be determined from the condition

$$
x^{\prime \prime}(0)+p x(0)+q x^{3}(0)=0
$$

and this condition is equivalent to

$$
q A^{4}+2 p A^{2}-\left(2 p x_{0}^{2}+q x_{0}^{4}+2 \dot{x}_{0}^{2}\right)=0
$$

The roots of equation (24) give the value of $A$ as follows:

$$
A= \pm \sqrt{\frac{-p \pm \sqrt{\Delta}}{q}}
$$

where $\Delta=\left(p+q x_{0}^{2}\right)^{2}+2 q \dot{x}_{0}^{2}$.

Remark 1. Solution (21) is periodic with period:

$$
T=2 \int_{e_{1}}^{\infty} \frac{\mathrm{d} x}{\sqrt{4 x^{3}-g_{2} x-g_{3}}},
$$

where $e_{1}$ is the greatest real root to the cubic

$$
4 z^{3}-g_{2} z-g_{3}=0 .
$$

3.2. An Analytical Solution of Quadratic Damping Duffing Equation in Terms of Weierstrass Elliptic Function. Here, to find an exact solution to the IVP (4) in terms of Weierstrass elliptic function, the following ansatz is introduced:

$$
x(t)=A+\frac{B}{1+C \wp(t)},
$$

where $\wp(t) \equiv \wp\left(t-t_{0} ; g_{2}, g_{3}\right)$.

Inserting ansatz (28) and equation (18) into $\ddot{x}+\varepsilon \dot{x}^{2}+p x+q x^{3}=0$, we get $R(t)=0$, with

$$
R(t)=\sum_{j=0}^{4} L_{j} \wp^{j}(t),
$$

where the values of the coefficients $L_{j}$ are given in Appendix B. Equating the coefficients $L_{j}$ to zero gives an algebraic equation system, and by solving this system, we obtain

$$
B=\frac{6 A\left(A^{2} q+p\right)}{2 A^{3} q \varepsilon-3 A^{2} q+2 A p \varepsilon-p},
$$

$$
C=\frac{-12}{2 A^{3} q \varepsilon-3 A^{2} q+2 A p \varepsilon-p},
$$




$$
\begin{aligned}
& g_{2}=\frac{1}{12}\left(\begin{array}{c}
-4 q^{2} \varepsilon^{2} A^{6}-q\left(8 p \varepsilon^{2}+3 q\right) A^{4} \\
-2 p\left(2 p \varepsilon^{2}+3 q\right) A^{2}+p^{2}
\end{array}\right), \\
& g_{3}=W_{1} \times W_{2},
\end{aligned}
$$

with

$$
\begin{gathered}
W_{1}=\frac{A^{3} q \varepsilon+A p \varepsilon+p}{216\left(5 A^{3} q \varepsilon-3 A^{2} q+5 A p \varepsilon-p\right)} \\
W_{2}=\left(\begin{array}{c}
8 q^{3} \varepsilon^{3} A^{9}-12 q^{3} \varepsilon^{2} A^{8} \\
+6 q^{2} \varepsilon\left(4 p \varepsilon^{2}+3 q\right) A^{7} \\
-9 q^{2}\left(4 p \varepsilon^{2}+3 q\right) A^{6} \\
+6 p q \varepsilon\left(4 p \varepsilon^{2}+11 q\right) A^{5} \\
-9 p q\left(4 p \varepsilon^{2}+7 q\right) A^{4}+ \\
2 p^{2} \varepsilon\left(4 p \varepsilon^{2}+27 q\right) A^{3} \\
-3 p^{2}\left(4 p \varepsilon^{2}+7 q\right) A^{2} \\
+6 p^{3} \varepsilon A-p^{3}
\end{array}\right) .
\end{gathered}
$$

The value of $A$ may be determined from the following cubic equation:

$$
\begin{aligned}
P \equiv & -3 q-8 p \varepsilon^{2} \\
& +4 \varepsilon\left(3 q+8 p \varepsilon^{2}\right) A-24 q \varepsilon^{2} A^{2}+32 q \varepsilon^{3} A^{3}=0
\end{aligned}
$$

We choose the first root of equation (35) as the default value to $A$ or any real root. Now, we must determine the value of $t_{0}$ so that

$$
\begin{aligned}
x(0) & =x_{0}, \\
x^{\prime}(0) & =\dot{x}_{0} .
\end{aligned}
$$

Let

$$
\left\{\begin{array}{l}
c_{0}=\wp\left(t_{0} ; g_{2}, g_{3}\right), \\
c_{1}=\wp^{\prime}\left(t_{0} ; g_{2}, g_{3}\right), \\
t_{0}=-\wp^{-1}\left(c_{0} ; g_{2}, g_{3}\right) .
\end{array}\right.
$$

Making use of the addition formula for the Weierstrass function gives

$$
x(t)=A+\frac{B}{1+C\left[(1 / 4)\left(\left(c_{1}-\wp\left(t ; g_{2}, g_{3}\right)\right) /\left(c_{0}-\wp\left(t ; g_{2}, g_{3}\right)\right)\right)^{2}-c_{0}-\wp\left(t ; g_{2}, g_{3}\right)\right]},
$$

where the values of $A, B$, and $C$ are defined in equations (30), (31), and (35).Using the initial conditions given in equation (36) will give us

$$
\begin{gathered}
A+\frac{B}{c_{0} C+1}=x_{0}, \\
-\frac{B c_{1} C}{\left(c_{0} C+1\right)^{2}}=\dot{x}_{0} .
\end{gathered}
$$

By solving system (39), we can get the values of $c_{0}$ and $c_{1}$ as

$$
\begin{aligned}
& c_{0}=\frac{x_{0}-A-B}{C\left(A-x_{0}\right)}, \\
& c_{1}=-\frac{B \dot{x}_{0}}{C\left(A-x_{0}\right)^{2}} .
\end{aligned}
$$

$$
\begin{aligned}
R(0)= & 0=2 q \varepsilon^{2} A^{6}-3 q \varepsilon\left(2 x_{0} \varepsilon-1\right) A^{5} \\
& +\left(2 p \varepsilon^{2}+6 q x_{0}^{2} \varepsilon^{2}-3 q x_{0} \varepsilon+3 q\right) A^{4} \\
& -\varepsilon\left(6 p x_{0} \varepsilon-5 p+2 q x_{0}^{3} \varepsilon+3 q x_{0}^{2}\right) A^{3} \\
& +3\left(2 p x_{0}^{2} \varepsilon^{2}-3 p x_{0} \varepsilon+2 p+q x_{0}^{3} \varepsilon\right) A^{2} \\
& -\varepsilon\left(2 p x_{0}^{3} \varepsilon-3 p x_{0}^{2}-3 \dot{x}_{0}^{2}\right) A \\
& +\left(p x_{0}^{3} \varepsilon-6 p x_{0}^{2}-3 q x_{0}^{4}-3 \dot{x}_{0}^{2} x_{0} \varepsilon-6 \dot{x}_{0}^{2}\right) .
\end{aligned}
$$

The resultant of the polynomials given in equations (35) and (41) with respect to $A$ reads

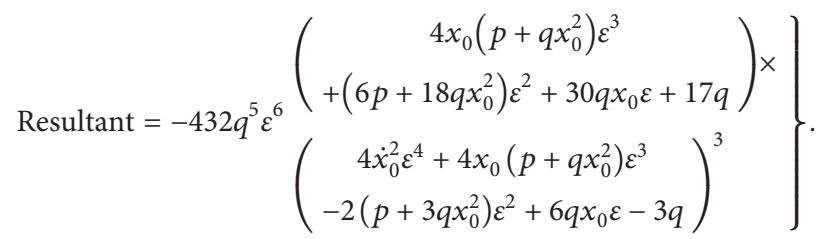

Note that the value of $A$ must also satisfy the condition 


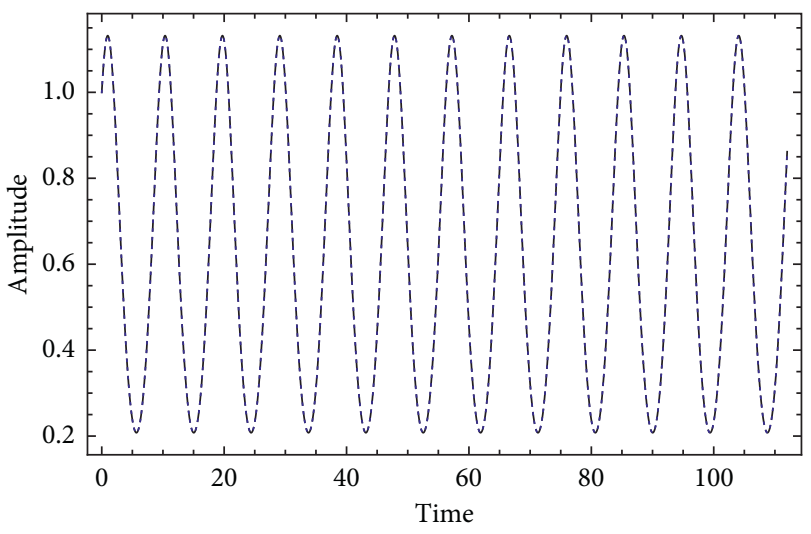

FIgURE 3: A comparison between the analytical solution (38) (dashed curve) and numerical solution using RK4 (dotted curve). Here, $p=-0.7, \varepsilon=0.1, x_{0}=1$, and $\dot{x}_{0}=0.25$.

This resultant must vanish in order to get exact solutions to the problem under consideration.

We may consider the condition

$$
4 \dot{x}_{0}^{2} \varepsilon^{4}+4 x_{0}\left(p+q x_{0}^{2}\right) \varepsilon^{3}-2\left(p+3 q x_{0}^{2}\right) \varepsilon^{2}+6 q x_{0} \varepsilon-3 q=0 .
$$

From this condition, we can calculate the value of $q$ as

$$
q=-\frac{2 \varepsilon^{2}\left(p\left(2 \varepsilon x_{0}-1\right)+2 \varepsilon^{2} \dot{x}_{0}^{2}\right)}{4 \varepsilon^{3} x_{0}^{3}-6 \varepsilon^{2} x_{0}^{2}+6 \varepsilon x_{0}-3} .
$$

Thus, the IVP (4) has been solved analytically under restriction (43) or (44).

A comparison between the analytical solution (38) and the approximate numerical RK4 solution is introduced in Figure 3. It is observed that our solution is in agreement with the numerical simulation solution.

In the limiting case, i.e., for $\varepsilon \longrightarrow 0$, the exact solution to the undamping Duffing problem (1) for arbitrary initial conditions may be covered

$$
x(t)=A-\frac{6 A\left(p+A^{2} q\right)}{p+3 A^{2} q+12 \wp\left(\begin{array}{c}
t-t_{0} ;(1 / 12)\left(-3 q^{2} A^{4}-6 p q A^{2}+p^{2}\right) \\
(1 / 216) p\left(9 q^{2} A^{4}+18 p q A^{2}+p^{2}\right)
\end{array}\right)}
$$

where

$$
\begin{aligned}
& t_{0}=\wp^{-1}\left(\begin{array}{l}
\frac{3 A^{3} q+3 A^{2} q x_{0}+5 p A+p x_{0}}{12\left(A-x_{0}\right)} ; \\
\frac{1}{12}\left(-3 q^{2} A^{4}-6 p q A^{2}+p^{2}\right), \\
\frac{1}{216} p\left(9 q^{2} A^{4}+18 p q A^{2}+p^{2}\right)
\end{array}\right), \\
& A= \pm \sqrt{\frac{-p \pm \sqrt{\left(p+q x_{0}^{2}\right)^{2}+2 q \dot{x}_{0}^{2}}}{q}}
\end{aligned}
$$

Note that both solutions (21) and (45) are equivalent.

\section{Conclusion}

The equation of motion with higher-order nonlinear restoring force and higher-order nonlinear damping force in which the damping is proportional to the square of the velocity has been carefully studied. As an example of this type of equation of motion, the stability analysis of the quadratic damping higher-order nonlinear Duffing oscillator equation using the Bifurcation method has been investigated. Then comes the main goal, which is to find some exact solutions to the Duffing oscillator equation and its family. In the form of Weierstrass elliptic function, the exact solution of the undamping higher-order nonlinear Duffing equation has been discussed. As for the second and most important goal of this study, it is to obtain an exact solution to the quadratic damping higher-order nonlinear Duffing oscillator equation. In general, the damping higher-order nonlinear Duffing oscillator equation is nonintegrable differential equation but under a certain condition it becomes integrable. Accordingly, the condition that makes this equation integrable has been deduced, and then, this solution has been obtained in detail in terms of the Weierstrass elliptic function. Furthermore, a comparison between the obtained solutions and the numerical RK4 solution to the Duffing equation and its family has been carried out. Also, in the limiting case, i.e., for $\varepsilon \longrightarrow 0$, the exact solution of the quadratic damping higher-order nonlinear Duffing oscillator equation has been reduced to the exact solution of the undamping higher-order nonlinear Duffing equation.

In future work, the Weierstrass elliptic functions will be devoted to find some exact and approximate analytical solutions to the forced higher-order damping cubic-quintic Duffing oscillator with external constant and periodic forces.

\section{Appendix}

\section{A. Equation (A.1)}

$$
\begin{aligned}
\mathbb{R}_{0}= & 2 A^{3} q+6 A^{2} B q+6 A B^{2} q+2 A p+2 B^{3} q-4 B C^{2} g_{3} \\
& +B C g_{2}+2 B p, \\
\mathbb{R}_{1}= & C\left(6 A^{3} q+12 A^{2} B q+6 A B^{2} q+6 A p-3 B C g_{2}+4 B p\right), \\
\mathbb{R}_{2}= & 2 C\left(3 A^{3} C q+3 A^{2} B C q+3 A C p+B C p-6 B\right), \\
\mathbb{R}_{3}= & 2 C^{2}\left(A^{3} C q+A C p+2 B\right) .
\end{aligned}
$$




\section{B. Equation (B.1)}

$$
\begin{aligned}
& L_{4}=2 C^{3}\left(A^{3} C q+A C p+2 B\right), \\
& L_{3}=2 C^{2}\left(\begin{array}{c}
4 A^{3} C q+3 A^{2} B C q \\
+4 A C p+4 B^{2} \varepsilon+B C p-4 B
\end{array}\right), \\
& L_{2}=3 C\left(\begin{array}{c}
4 A^{3} C q+6 A^{2} B C q+2 A B^{2} C q \\
+4 A C p-B C^{2} g_{2}+2 B C p-4 B
\end{array}\right), \\
& L_{1}=2 C\left(\begin{array}{c}
4 A^{3} q+9 A^{2} B q+6 A B^{2} q+4 A p+B^{3} q \\
-B^{2} C g_{2} \varepsilon-2 B C^{2} g_{3}-B C g_{2}+3 B p
\end{array}\right), \\
& L_{0}=\left(\begin{array}{c}
2 A^{3} q+6 A^{2} B q+6 A B^{2} q+2 A p+2 B^{3} q \\
-2 B^{2} C^{2} g_{3} \varepsilon-4 B C^{2} g_{3}+B C g_{2}+2 B p
\end{array}\right) .
\end{aligned}
$$

\section{Data Availability}

The datasets generated for this paper are available from the corresponding author upon request.

\section{Conflicts of Interest}

The authors declare that they have no conflicts of interest.

\section{Acknowledgments}

The authors acknowledge the Deanship of Scientific Research (DSR) in King Abdulaziz University for its support.

\section{References}

[1] A. M. Wazwaz, Partial Differential Equations and Solitary Waves Theory, Higher Education Press, Beijing, China, 2009.

[2] A. M. Wazwaz, Partial Differential Equations: Methods and Applications, Balkema, cop, Lisse, Netherlands, 2002.

[3] M. B. Arain, M. M. Bhatti, A. Zeeshan, T. Saeed, and A. Hobiny, "Analysis of arrhenius kinetics on multiphase flow between a pair of rotating circular plates," Mathematical Problems in Engineering, vol. 2020, Article ID 2749105, 17 pages, 2020.

[4] M. M. Bhatti and I. Sara, "Abdelsalam, thermodynamic entropy of a magnetized Ree-Eyring particle-fluid motion with irreversibility process: a mathematical paradigm," Zeitschrift für Angewandte Mathematik und Mechanik, Article ID e202000186, 2020.

[5] A. H. Salas and S. A. El-Tantawy, "On the approximate solutions to a damped harmonic oscillator with higher-order nonlinearities and its application to plasma physics: semianalytical solution and moving boundary method," The European Physical Journal Plus, vol. 135, pp. 833-917, 2020.

[6] M. Abu Hammad, A. H. Salas, and S. A. El-Tantawy, "New method for solving strong conservative odd parity nonlinear oscillators: applications to plasma physics and rigid rotator," AIP Advances, vol. 10, Article ID 085001, 11 pages, 2020.

[7] J. Guckenheimer and P. Holmes, Nonlinear Oscillations, Dynamical Systems and Bifurcations of Vector Fields, Springer-Verlag, Heidelberg, Germany, 1983.
[8] F. Bakhtiari-Nejad and M. Nazari, "Nonlinear vibration analysis of isotropic cantilever plate with viscoelastic laminate," Nonlinear Dynamics, vol. 56, no. 4, pp. 325-356, 2009.

[9] N. Srinil and H. Zanganeh, "Modelling of coupled cross-flow/ in-line vortex-induced vibrations using double Duffing and van der pol oscillators," Ocean Engineering, vol. 53, pp. 83-97, 2012.

[10] D. Younesian, H. Askari, Z. Saadatnia, and M. K. Yazdi, "Periodic solutions for nonlinear oscillation of a centrifugal governor system using the he's frequency-amplitude formulation and he's energy balance method," Nonlinear Science Letters A, vol. 2, pp. 143-148, 2011.

[11] I. Kovacic and M. J. Brennan, The Duffing Equation: Nonlinear Oscillators and Their Behaviour, John Wiley \& Sons, Ltd., Hoboken, NJ, USA, 1st edition, 2011.

[12] P. S. Landa, Nonlinear Oscillations and Waves in Dynamical Systems, Springer, Heidelberg, Germany, 1996.

[13] U. R. Singh and G. Purohit, "Synchronization of quadratically damped mathieu- duffing based chaotic circuits with applications to secure communications," International Journal of Science and Research, vol. 5, pp. 80-85, 2016.

[14] U. R. Singh, G. Purohit, A. Sharma, and V. Patidar, "An analogue circuit to study the forced and quadratically damped Duffing oscillator," International Journal of Nonlinear Dynamics and Control, vol. 1, no. 1, pp. 87-96, 2017.

[15] R. E. Mickens, "Mathematical and numerical study of the duffing-harmonic oscillator," Journal of Sound and Vibration, vol. 244, no. 3, pp. 563-567, 2001.

[16] J. H. He, "Comment on he's frequency formulation for nonlinear oscillators," European Journal of Physics, vol. 29, pp. 19-22, 2008.

[17] J.-H. He, "Homotopy perturbation technique," Computer Methods in Applied Mechanics and Engineering, vol. 178, no. 3-4, pp. 257-262, 1999.

[18] Y. Khan, M. Akbarzade, and A. Kargar, "Coupling of homotopy and the variational approach for a conservative oscillator with strong odd-nonlinearity," Scientia Iranica, vol. 19, no. 3, pp. 417-422, 2012.

[19] M. El-Shahed, "Application of differential transform method to non-linear oscillatory systems," Communications in Nonlinear Science and Numerical Simulation, vol. 13, pp. 17141720, 2008.

[20] B. S. Wu and W. P. Sun, "Construction of approximate analytical solutions to strongly nonlinear damped oscillators," Archive of Applied Mechanics, vol. 81, no. 8, pp. 1017-1030, 2011.

[21] L. Cveticanin, "Oscillators with nonlinear elastic and damping forces," Computers \& Mathematics with Applications, vol. 62, no. 4, pp. 1745-1757, 2011.

[22] A. Y. T. Leung, Z. Guo, and H. X. Yang, "Residue harmonic balance analysis for the damped duffing resonator driven by a van der pol oscillator," International Journal of Mechanical Sciences, vol. 63, no. 1, pp. 59-65, 2012.

[23] M. Turkyilmazoglu, "An effective approach for approximate analytical solutions of the damped duffing equation," Physica Scripta, vol. 86, no. 6, Article ID 015301, 2012.

[24] Y. Khan and Q. Wu, "Homotopy perturbation transform method for nonlinear equations using he's polynomials," Computers \& Mathematics with Applications, vol. 61, pp. 1963-1967, 2011.

[25] Y. Khan and F. Austin, "Application of the laplace decomposition method to nonlinear homogeneous and non-homogenous advection equations," Zeitschrift für Naturforschung A, vol. 65, no. 10, pp. 849-853, 2010. 
[26] S. Nourazar and A. Mirzabeigy, "Approximate solution for nonlinear duffing oscillator with damping effect using the modified differential transform method," Scientia Iranica, vol. 20, pp. 364-368, 2013.

[27] J.-H. He, "Preliminary report on the energy balance for nonlinear oscillations," Mechanics Research Communications, vol. 29, no. 2-3, pp. 107-111, 2002.

[28] S. Durmaz, S. A. Demirbag, and M. O. Kaya, "High order he's energy balance method based on collocation method," International Journal of Nonlinear Sciences and Numerical Simulation, vol. 11, no. 1-5, 2010.

[29] M. K. Yazdi, H. Ahmadian, A. Mirzabeigy, and A. Yildirim, "Dynamic analysis of vibrating systems with nonlinearities," Communications in Theoretical Physics, vol. 57, no. 2, pp. 183-187, 2012.

[30] A. Elias-Zungia, "Analytical solution of the damped helmholtz-duffing equation," Applied Mathematics Letters, vol. 25, pp. 2349-2353, 2012.

[31] Y. Geng, "Exact solutions for the quadratic mixed-parity helmholtz-duffing oscillator by bifurcation theory of dynamical systems," Chaos, Solitons and Fractals, vol. 81, pp. $68-77,2018$.

[32] E. T. Whittaker and G. N. Watson, A Course of Modern Analysis, Cambridge University Press, Cambridge, UK, 4th edition, 1980.

[33] M. Abramowitz and I. Stegun, Handbook of Mathematical Functions, Dover, New York NY, USA, 9th edition, 1980.

[34] K. Johannesen, "The duffing oscillator with damping," European Journal of Physics, vol. 36, no. 13, Article ID 065020, 2015.

[35] K. Johannesen, "The duffing oscillator with damping for a softening potential," International Journal of Applied and Computational Mathematics, vol. 3, pp. 3805-3816, 2017.

[36] J.-w. Zhu, "A new exact solution of a damped quadratic nonlinear oscillator," Applied Mathematical Modelling, vol. 38, no. 24, pp. 5986-5993, 2014.

[37] B. Baumann, J. Schwieger, M. Wolff, F. Manders, and J. Suijker, "Nonlinear behavior in high-intensity discharge lamps," Journal of Physics D Applied Physics, vol. 49, Article ID 255201, 2015.

[38] L. Cveticanin, "Oscillator with strong quadratic damping force," Publications de l'Institut Math?matique (Belgrade), vol. 85, no. 99, pp. 119-130, 2009.

[39] R. Porwal and N. S. Vyas, "Damped quadratic and mixedparity oscillator response using krylov-bogoliubov method and energy balance," Journal of Sound and Vibration, vol. 309, no. 3-5, pp. 877-886, 2008.

[40] S. K. Lai and K. W. Chow, "Exact solutions for oscillators with quadratic damping and mixed-parity nonlinearity," Physica Scripta, vol. 85, Article ID 045006, 2012.

[41] B. R. Smith, "The quadratically damped oscillator: a case study of a non-linear equation of motion," American Journal of Physics, vol. 80, no. 9, p. 816, 2012. 